\title{
Distintas familias, distintos consumos: relación de las dinámicas familiares con el consumo de alcohol en adolescentes en Colombia*
}

\author{
Juliana Mejía-Trujillo**
}

Recibido: 11 de julio de 2016

Evaluado: 12 de septiembre de 2016

Aceptado: 2 de octubre de 2016

\section{Resumen}

La mayoría de estudios sobre consumo de alcohol en adolescentes se limita a la presentación de estadísticas sobre prevalencias. Este estudio se centra en las dimensiones cualitativas del problema, por medio de la realización de diez grupos focales; cinco con padres de familia y cinco con adolescentes, con un total de 116 personas. Se llevaron a cabo en Bogotá, Barranquilla, Bucaramanga, Cali y Medellín. Los resultados muestran que el consumo de alcohol en menores de edad es un hecho normalizado por la cultura; que los primeros consumos casi siempre ocurren en reuniones familiares; que las dinámicas en las familias relacionadas con normas, límites, autoridad, socialización y relaciones son fundamentales para los adolescentes a la hora de tomar la decisión de ingerir alcohol o no, más que el conocimiento que tienen de los riesgos; y que los programas preventivos deben ir principalmente dirigidos al ámbito familiar, y no tanto a los mismos adolescentes, ya que en muchos casos el consumo es inducido por los miembros mayores de la familia, como abuelos, tíos y, en ocasiones, por los mismos padres.

Palabras clave: alcohol, adolescentes, patrones de consumo, dinámicas familiares, investigación cualitativa. 


\section{Different Families, Different Consumption Patterns: Relationship Between Family Dynamics and Alcohol Consumption among Adolescents in Colombia}

\begin{abstract}
The majority of studies on adolescents' alcohol use are limited to presenting prevalences of use. This study is centered on the qualitative dimensions of the problem through the implementation of 10 focus groups, five carried out with parents and another five with adolescents in Bogotá, Barranquilla, Bucaramanga, Cali and Medellín. Results show that alcohol consumption among minors is a fact normalized culturally; that family dynamics related to norms, limits, authority, socialization and relationships, are fundamental for adolescents at the time of deciding on the convenience of drinking or not, more than the knowledge about social and health risks; and that preventive programs should be mainly directed to the family domain, and not to the youngsters, because in many cases alcohol consumption is induced by
\end{abstract}

Keywords: Alcohol, adolescents, consumption patterns, family dynamics, qualitative research.
Received: July 11, 2016

Evaluated: September 12, 2016

Accepted: October 2, 2016 


\section{Distintas famílias, distintos consumos: relação das dinâmicas familiares com o consumo de álcool dos adolescentes na Colômbia}

Recebido: 11 de julho de 2016

Avaliada: 12 de setembro de 2016

Aceito: 2 de outubro de 2016

\section{Resumo}

A maioria dos estudos sobre o consumo de bebida alcoólica na adolescência se limita à apresentação de estatísticas de prevalência. Este estudo enfoca as dimensões qualitativas do problema, através da realização de dez grupos focais; cinco com pais e cinco com adolescentes, totalizando 116 participantes. Foram realizadas em Bogotá, Barranquilla, Bucaramanga, Cali e Medellín. Os resultados mostram que o consumo de álcool em menores de idade é um fato normalizado pela cultura; que o primeiro consumo quase sempre ocorre em encontros familiares; que as dinâmicas das famílias relacionadas às normas, limites, autoridade, socialização e relacionamentos são fundamentais para os adolescentes quando tomam a decisão de beber álcool ou não, ao invés do conhecimento que possuem dos riscos; e que os programas preventivos devem ser direcionados principalmente para a família, e não tanto para os próprios adolescentes, já que em muitos casos o consumo é induzido pelos membros mais velhos da família, como avós, tios e, às vezes, pelo mesmo pais.

Palavras-chave: álcool, adolescentes, padrões de consumo, dinâmica familiar, pesquisa qualitativa. 


\section{INTRODUCCIÓN}

Tomar un trago en nuestra sociedad puede ser considerado como un acto banal o cotidiano. Sin embargo, cuando se indaga sobre los usos culturales y sociales de lo que significa beber sustancias alcohólicas, la forma de entender este consumo puede ser diferente, más aún cuando se busca entenderlo dentro de un grupo social, como el de los adolescentes. El consumo de alcohol por parte de menores de edad se inicia principalmente en los propios hogares, con la aquiescencia de las mismas familias; así, de manera indirecta, el consumo de alcohol por parte de adolescentes es un comportamiento socialmente aceptado (Yoon, Lam, Sham, \& Lam, 2015).

Los valores culturales que promueven el consumo de esta sustancia en la población que aún no ha cumplido la mayoría de edad son transmitidos por socialización familiar, en primera medida, y por pares, en segunda instancia; en ambos casos, las normas, los valores aprendidos y las ideas personales desempeñan un papel determinante en la decisión de tomar o no. Los daños causados en el cerebro de los adolescentes, por no haber terminado su desarrollo, son una de las principales razones para buscar que ellos no consuman alcohol antes de la edad legal permitida (Pascual, Pla, Miñarro \& Guerri, 2014; Ward, Lallemand, \& De Witte, 2014); sin embargo, otros riesgos que conlleva el consumo, como la exposición a situaciones de violencia, accidentes de tránsito (Schofield \& Denson, 2013), embarazo adolescente, infecciones de transmisión sexual (Stickley et al., 2013) y fracaso escolar (Briones \& Woods, 2013), se constituyen en un problema social, por las edades tempranas en las que ocurren, por ello debe ser abordado desde distintas perspectivas, entre ellas la antropológica.

El último estudio nacional de consumo de sustancias psicoactivas en escolares (Ministerio de Justicia y el Derecho, et al. 2011) indica que el $63 \%$ de los estudiantes de $6 .^{\circ}$ a $11 .^{\circ}$, de colegios públicos y privados en $\mathrm{Co}-$ lombia, ha consumido alcohol alguna vez en su vida; el $57 \%$ lo hizo en el último año, y el $40 \%$, en el último mes. Esta última prevalencia sugiere un consumo habitual de alcohol. Sin embargo, al observar las estadísticas y los factores asociados con el consumo de alcohol y sustancias psicoactivas, son reiterativos y en muchos casos determinantes los factores de riesgo relacionados con la familia, no solo por el escaso control parental, sino, también, por las actitudes favorables de los padres hacia el consumo, las relaciones parentofiliales poco satisfactorias y el consumo por parte de hermanos mayores (Arthur, Hawkins, Pollard, Catalano, \& Baglioni Jr., 2002; Fagan, Van Horn, Hawkins, \& Arthur, 2007; Gossrau-Breen, Kuntsche, \& Gmel, 2010).

Con base en la literatura sobre el tema y un trabajo exploratorio con un grupo de jóvenes, este artículo pretende analizar la relación de las dinámicas familiares vinculadas con el consumo de alcohol, y su influencia en las experiencias de consumo de los adolescentes.

\section{LA BEBIDA}

El alcohol es una bebida tan antigua como la humanidad, cuyos modos de producción, comercialización, usos y patrones han cambiado a lo largo de los años y las culturas. 
Los primeros registros datan del 7000 a. C., en China; allí se obtenían bebidas alcohólicas por fermentación (Guelbenzu, 2011); también se conocen bebidas de arroz, frutas y miel en India, Babilonia, Egipto, entre otros (Lyman, 2011; Vaillant, 1983). En América Latina también fue y sigue siendo popular el uso de bebidas por fermentación para embriagarse, aunque inicialmente solo tenían un uso ritual (Pérez-Gómez, 1986).

El alcohol es una sustancia depresora del sistema nervioso central (SNC), razón por la cual genera un decremento en el grado de conciencia y en la capacidad de respuesta sensorial, disminución de las funciones cognitivas y problemas en la ejecución de actividades físicas; estas consecuencias se dan en menor o mayor grado, de acuerdo con la edad, la cantidad y la frecuencia del consumo de alcohol (Pérez-Gómez, Ruiz-Giraldo, Villar-Concha \& Uribe-Martínez, 2000; Stimson, 2006). Sin embargo, lo clínico confrontado con lo cultural plantea una complejidad analítica al momento de entender las lógicas de consumo: por ejemplo, con los patrones seguidos en algunos países europeos, como Francia, donde es común tomar una copa de vino diaria acompañada de la comida, incluso desde la adolescencia, lo que sería una práctica opuesta a lo expresado desde el punto de vista biomédico.

Los efectos pueden exacerbarse y afectar el desarrollo cuando quienes consumen son niños y adolescentes (Stimson, 2006), pues la corteza prefrontal y el hipocampo (las partes que más se afectan por el alcohol) no se desarrollan completamente sino hasta los 21 años de edad, y son las encargadas de gran parte de las funciones cognitivas superiores (toma de decisiones, planeación, raciocinio, control de impulsos, memoria, abstracción y aprendizaje). Además, quienes comienzan a consumir alcohol antes de los 14 años de edad tienen hasta diez veces más de probabilidades de tener problemas con el alcohol en la adultez o de consumir otras sustancias, que quienes empiezan a tomar después de los 18 años (Pérez-Gómez, Scoppetta, \& Flórez-Alarcón, 2011); a todo ello se agregan los problemas sociales asociados con la mortalidad juvenil por consumo de alcohol, debido a accidentes de tránsito, suicidio y situaciones de violencia y crimen (Schofield et al. 2013).

Las causas para iniciar el consumo de alcohol pueden ser múltiples, pero al hacer una revisión de la literatura, es reiterativo que los factores de riesgo familiares tienen una alta incidencia: las actitudes favorables de los padres hacia el consumo de alcohol son un factor de riesgo muy estudiado, que en conjunto con el escaso control parental, constituyen las principales causas de consumo de alcohol en adolescentes (Arthur et al., 2002; Fagan et al., 2007). Una actitud favorable hace referencia a la valoración del alcohol como algo positivo para la propia persona, y puede convertirse en un predictor del consumo, ya que a esta actitud se puede llegar por modelamiento o ejemplo de los padres o familiares, así como por el grupo de pares, quienes también podrían aumentar la probabilidad del consumo de los adolescentes (Becoña, 2002).

Flórez y Trujillo (2013) encontraron que los motivos de consumo o no de alcohol de los adolescentes reflejan la cultura de consumo de la comunidad a la que pertenecen; así, sostienen que la decisión de consumir o no por parte de los adolescentes 
depende en gran medida de la situación en la que se encuentren, y no necesariamente del proceso reflexivo en torno a los riesgos que conlleva el consumo de la sustancia. Otros elementos definitivos para tomar la decisión fueron la imagen y el prejuicio social que el adolescente tiene del consumidor de alcohol, acompañada de la personalidad, la historia personal, las estrategias de afrontamiento y el contexto en el que se desenvuelve; por eso, una misma razón puede servir de motivación para tomar cualquiera de las dos decisiones (consumir o no consumir), lo cual confirma lo expresado por Kloep, Ngerbrigsten, Glendinning y Espness (2001), quienes sostienen que el consumo de alcohol es reconocido como un objeto de identificación e integración grupal, por lo cual lo común es que el consumo de alcohol sea una actividad social. Igualmente, Yoon et al. (2015) mencionan que en China beber alcohol es una actividad inherentemente social, y que la mayoría de las veces ocurre en el contexto de la familia y de las redes sociales.

Los diferentes grupos sociales y sus diversas culturas muestran que las dinámicas propias de las familias y de los entornos también contribuyen a fortalecer o a denigrar la percepción que tienen los adolescentes sobre el consumo de alcohol (Rolando, Beccaria, Tigerstedt \& Törrönenet, 2012); por eso, la ingesta de esta sustancia puede estar ligada con valores familiares, características interiorizadas en determinadas culturas, adopción de patrones de consumo de alcohol similares a otros miembros de la familia, presencia de la conducta en pares, menor percepción de riesgo, y cambios generacionales en los procesos de disciplina y socialización (Gmel, Kuntsche, \& Rehm,
2011; Valentine, Holloway, \& Jayne, 2010). Esto último se entiende como la manera mediante la cual un grupo social enseña las normas, los valores y las creencias a sus miembros, en distintas etapas del desarrollo (Campos, 2007).

Ligadas con estas razones se encuentran las creencias, reconocidas como formas de conocimiento que construyen ideologías simplificadas del mundo y de sus procesos, y representan aspectos de la realidad, que generalmente no pueden ser comprobados; las creencias positivas que tienen los padres frente a la ingesta de alcohol son uno de los aspectos que predicen la intención de consumo en el adolescente (Becoña, 2002), y hasta cierto punto condicionan el comportamiento con la bebida, al provocar la acogida o rechazo a la sustancia, justifican el uso del licor y hablan de los entornos en los que se desenvuelve el joven (Sierra, Pérez, Pérez y Muñoz, 2005).

Algunas creencias relacionadas con el consumo de alcohol es que facilita las relaciones sociales, porque genera facilidad en la expresión de sentimientos y desinhibición de la conducta, produce relajación física y psicológica, y la aceptación por el grupo de pares (Mora y Natera, 2001). Sin embargo, otras creencias incluyen los aspectos negativos del consumo, como los riesgos y efectos a corto, mediano y largo plazos, que pueden ser atribuidos a los peligros para la salud o sociales (Sierra et al., 2005).

El estudio realizado por Gossrau-Breen, Kuntsche y Gmel (2010) sobre el consumo de alcohol en menores de edad mostró que existe una gran relación con las relaciones parentofiliales satisfactorias, con el alto control parental y con el consumo de hermanos 
mayores; el efecto de esto último fue más determinante para consumir alcohol que las relaciones con los padres y que el mismo establecimiento de normas en el hogar respecto a dicha situación. Khoddam, Worley, Browne, Doran y Brown (2015) mencionaron que la historia de consumo familiar es predictiva del consumo de adolescentes, y el riesgo aumenta con el tiempo, especialmente en cantidad y frecuencia.

Otros aspectos relacionados con el involucramiento familiar asociado con el consumo de alcohol de los adolescentes sugieren que a mayor número de expendios de alcohol en los barrios, mayor es el número de padres que suministran alcohol a sus hijos; de esta manera incrementa la disponibilidad de la sustancia y así, la prevalencia de consumo de alcohol de último mes (Rowland, Toumbourou, Satyen, Livingston, \& Williams, 2014).

En un estudio cualitativo (Rolando, Beccaria, Tigerstedt \& Törrönenet, 2012), se presentan grandes diferencias entre dos países europeos respecto a las razones por las que las personas toman alcohol la primera vez; así, en Italia, el primer consumo de alcohol ha estado asociado con la familia, como un valor y un rasgo por seguir, mientras que en Finlandia lo recuerdan como una experiencia desagradable, ligada con la intoxicación, no con valores o principios familiares. Pese a esto, otros estudios muestran la preocupación creciente en países como Francia y la misma Italia por el incremento del consumo de alcohol en adolescentes (Gmel et al., 2011).

Estos hechos no son nuevos. En el Reino Unido se encontró que los jóvenes adoptan patrones de consumo de alcohol similares a los de sus padres en su propia juventud, pero actualmente consumen muchas más unidades de alcohol de lo que se bebía hace 30 años. En el estudio, esto se atribuye a distintas motivaciones y a que la presión de pares es más fuerte en la actualidad. No solo se observan cambios en la cantidad, sino, también, relacionados con el sexo, la disciplina, los procesos de socialización, la percepción de riesgo, las expectativas frente a los ciclos vitales, entre otros (Valentine et al., 2010), los cuales promueven un mayor consumo en el momento actual, comparado con lo que se consumía a mediados de los años ochenta.

Sin embargo, a pesar de contar con una amplia literatura, que asegura que el consumo de alcohol familiar podría incidir en el consumo adolescente, hay otros estudios que sugieren lo contrario; uno de ellos afirma que el riesgo es más alto cuando son gemelos o amigos, y hay un riesgo menor con otros familiares, como hermanos (no gemelos) o padres y madres; al estudiar a estos últimos de manera separada y no bajo la categoría de padres (Scholte, Poelen, Willemsen, Boomsma, \& Engels, 2008).

Además, la familia también desempeña un doble rol: aunque fomenta el consumo de alcohol (por medio de la socialización en el uso de esta $u$ otras sustancias por parte de los padres, o con actitudes favorables), también es la solución, por medio de la terapia familiar. Aunque se debe tener en cuenta que la familia no es la unidad primaria de socialización directa del uso de drogas y alcohol, sino que, más bien, son los atributos de la familia los que lo promueven. Tales atributos pueden ser la estructura, el apego y la actividad familiar; la primera hace 
referencia al vínculo de las personas que viven bajo el mismo techo; la segunda se refiere al grado de afecto e involucramiento entre padres e hijos, o, por el contrario, el grado de hostilidad en sus relaciones; la última se relaciona con el consumo de los padres, las actitudes y las normas que se establecen (Barker \& Hunt, 2004).

Las relaciones y vínculos establecidos entre los miembros de un grupo social que convive bajo un mismo techo y que se denomina a sí mismo familia hacen parte de las dinámicas familiares, al igual que los procesos de colaboración, conflicto, socialización, autoridad, afecto y límites (Torres, Ortega, Garrido \& Reyes, 2008). Estos procesos desempeñan un papel preponderante en la relación que establecen los adolescentes con el alcohol, pues la manera como cada uno de dichos procesos se desarrolle, puede promover o frenar su consumo. Este estudio permitió indagar sobre diversos elementos de la cultura, implícitos en las dinámicas familiares, sociales y personales, que promueven $\mathrm{u}$ obstaculizan el consumo de alcohol en menores de edad.

\section{Metodología}

A raíz de los resultados obtenidos en el estudio de alcohol en menores de edad en nueve municipios de Colombia, realizado por la Corporación Nuevos Rumbos (Pérez-Gómez \& Scoppetta, 2009), la preocupación por distintos aspectos familiares que promueven el consumo de alcohol en adolescentes llevó al planteamiento de diversas preguntas, que solo podían ser respondidas al poner la lupa en algunas características de las dinámicas familiares. La mejor manera de obtener esas respuestas fue por medio del desarrollo de un estudio cualitativo que diera cuenta de los rasgos de cinco regiones colombianas, en el marco del segundo estudio de alcohol en menores (Pérez, Mejía, Reyes \& Cardozo, 2015), que fue llevado a cabo en las mismas ciudades que el primero, con condiciones de población similares, para poder establecer la comparación.

Los resultados del aspecto cualitativo de dicho estudio se presentan en este artículo, el cual es de tipo antropológico, cualitativo, y muestra aspectos culturales del consumo de alcohol familiar y de los adolescentes; no tanto los aspectos individuales, sino aquellos ligados con las interacciones humanas. Los instrumentos utilizados para la recolección de la información fueron diálogos informales y grupos focales (Bernand, 2011), que permitieron ampliar la población en número y en variedad cultural.

Entre los meses de junio y agosto de 2015 se realizaron diez grupos focales, en cinco ciudades de Colombia (Barranquilla, Bogotá, Bucaramanga, Cali y Medellín); dos en cada ciudad, divididos por adultos y adolescentes, vinculados con los grados décimo y undécimo de instituciones educativas públicas, de estratos socioeconómicos dos y tres. La muestra fue de 116 participantes; el $64 \%$ eran mujeres y el $36 \%$ restante, hombres. Si bien se intentó equilibrar la participación en un mismo número de hombres y mujeres, no fue posible lograrlo en los grupos focales de adultos, en que la asistencia de mujeres fue siempre mayor que la de los hombres (87 y $13 \%$, respectivamente), mientras que los grupos focales de adolescentes se acercaron más al equilibrio de sexo (55\% hombres y $45 \%$ mujeres). No hubo diferencias respecto a grado escolar de los adolescentes; el 
$51 \%$ pertenecía a décimo grado, y el $49 \%$, a undécimo. Las edades de los jóvenes oscilaron entre los 15 y 18 años, mientras que las de los adultos superaban, en la mayoría de los casos, los 30 años, y no excedían los 60 .

Dichos grupos focales revelaron aspectos importantes en cuanto a dinámicas familiares, imaginarios sobre el consumo de alcohol, y normas o valores que obstaculizan o promueven el consumo en menores de edad. En estas mismas categorías se organizaron los hallazgos. Al momento de hacer el análisis fueron tenidas en cuenta tanto la perspectiva de los padres como la de los estudiantes; ambas tienen puntos en común, pero en un buen número discrepan. Las diferencias por ciudades en la mayoría de categorías no fueron notorias, pero cuando se evidenciaron, quedaron expresadas en los resultados.

Para la realización de los grupos focales se estableció contacto con directivos de las instituciones educativas o las secretarías de Educación de los municipios, quienes en un primer momento autorizaron la ejecución; posteriormente se solicitó consentimiento informado a los participantes adultos y firma de los padres para quienes eran menores de edad. Cada uno de los grupos focales tuvo una duración aproximada de una hora, fue grabado en audio y transcrito para su posterior categorización y análisis, por medio del programa Atlas Ti.

\section{Resultados}

La recolección y análisis de la información permitió entender el consumo de alcohol como un hecho social inmerso en la cultura colombiana, que permea la vida cotidiana de sus habitantes y afecta a los adolescentes en la medida en que los primeros tragos se consumen en el contexto familiar, según los hallazgos, a pesar de reconocer riesgos y consecuencias negativas. Así mismo, permitió reconocer imaginarios por parte de adultos y menores, que perpetúan esta práctica por generaciones como una situación normalizada de la cultura.

\section{Distintas familias, distintos consumos: dinámicas propias de socialización}

La familia es la unidad primaria de socialización en la que el niño desarrolla habilidades de interacción y pautas de comportamiento con las que se va a enfrentar al mundo adulto; así mismo, allí aprende las costumbres, valores, ritos y demás elementos familiares que le otorgan una identidad cultural. El consumo de alcohol es una de las acciones que se realizan por medio del seguimiento de procesos de ejemplificación de la familia, por eso es tan importante, al momento de hablar de las dinámicas familiares, reconocer cuáles de ellas promueven u obstaculizan el consumo de alcohol en la adolescencia.

Esta categoría hace referencia a la identificación de antecedentes familiares de consumo, ya sea en el núcleo o en la familia extensa, pues los adolescentes o los padres lo consideran como un referente, o consideran que su consumo ha ejercido influencia en otros miembros de la familia; también, hace referencia a las actitudes de padres y adolescentes frente al consumo de alcohol y a las situaciones de uso de la sustancia, asociadas con la familia. Algunos padres indicaron que les gusta el alcohol y que es una tradición familiar; en el estudio, esta respuesta se puede ver con mayor frecuencia 
en Barranquilla y Medellín. Los adolescentes confirmaron la larga y fuerte historia de consumo de alcohol en las familias en general, pero indicaron que el padre ha sido quien más lo ha usado, seguido de la familia extensa.

Mi familia es demasiado alcohólica cuando hacen eventos. (Grupo focal de adolescentes, Cali)

\section{$[\ldots]$}

Creo que en mi casa por parte de papá no hay fin de semana que no tomen, $y$ mientras más gente haya mucho mejor, hasta que no ven a alguien en la inmunda no paran de tomar, y jay de los que no tomen! (Grupo focal de adolescentes, Bogotá)

\section{$[\ldots]$}

Mi papá toma toda la semana, mi papá es alcohólico y yo hablo con él, pero no por eso yo voy a hacer lo mismo, si uno ve que el papá es alcohólico, uno no se va a poner en esas. (Grupo focal de adolescentes, Barranquilla)

Los adultos también manifestaron que se han generado cambios en los patrones de consumo de alcohol propios, a raíz de diversas experiencias negativas con la bebida, ya sean de su familia de origen, extensa o de personas cercanas. Se refirieron al alcohol como algo desagradable, por lo que han disminuido frecuencia y cantidad de consumo, hasta alcanzar, en algunos casos, el alejamiento total del licor o el abandono de personas que lo hacían, especialmente sus propias parejas. Bogotá y Cali fueron las ciudades en las que fue más común esta situación.
Por generaciones a mi familia le ha gustado, les ha gustado el trago en mi casa. (Grupo focal de padres, Barranquilla)

$[\ldots]$

Por ejemplo, mi esposo detesta el alcohol porque el papá era muy tomatrago, duraba una y hasta tres semanas tomando y tuvo un accidente borracho. (Grupo focal de padres, Cali)

$[\ldots]$

El papá de mi hijo tomaba mucho y por eso me separe de él, de verdad. (Grupo focal de padres, Bogotá)

Los adolescentes estuvieron de acuerdo con lo expresado por los padres: asociaron las experiencias del consumo de alcohol de los adultos con oportunidades para disminuir el consumo o para dejar de tomar por completo. Incluso, los adolescentes fueron más específicos que los adultos al describir las situaciones en las que se ha generado disminución de consumo de alcohol; por ejemplo, accidentes en los que hicieron daño a otros.

Yo tenía por ahí 8 años cuando papá tomaba [...] pero dejó de tomar por un tiempo cuando mi hermana nació, porque un día estábamos en la casa de mi tía, estaba tomado y quería cargar a mi hermana recién nacida y mi mamá dijo que no, pero él la quería cargar y estábamos en un tercer piso [...] él se resbaló y botó a mi hermana por el balcón, y pues cosas de la vida que cayó sobre una matera y no le pasó nada, entonces después de ese susto, mi papá dijo que no se volvía a tomar ni una sola cerveza. (Grupo focal de adolescentes, Cali) 
Las dinámicas familiares desempeñan un rol muy importante en la percepción que tienen los adolescentes sobre el alcohol. Se pudo observar que las historias familiares han generado cambios hacia la disminución del consumo de los padres, especialmente; pero en el conjunto de la familia extensa, ha continuado la frecuencia y la cantidad de alcohol a lo largo del tiempo, hecho que en ocasiones parece ser motivo de orgullo y requisito para sentirse parte de la familia; en otras, esta trayectoria de consumo sirve como un ejemplo que los adolescentes no desean seguir.

Las familias, de manera tácita o expresa, han enseñado a sus hijos que consumir en algún momento de la vida es algo común y legítimo en la cultura colombiana, por lo cual los adolescentes aprenden que entre más jóvenes lo hagan, más vinculados están con la cultura, ya que los adultos por lo general expresan que en el pasado bebían mayor cantidad de licor.

\section{Palabras y hechos que incitan a tomar}

La percepción positiva de padres y adolescentes sobre el consumo de alcohol en menores de edad fue tomada por estos últimos como una posibilidad de ingerir alcohol desde edades tempranas; la actitud positiva de los padres ha reforzado los comportamientos de los jóvenes frente a la bebida, especialmente si la actitud ha estado acompañada del comportamiento de consumo. La verbalización de la actitud frente al alcohol no es un hecho simple o sencillo de realizar, debido a que hay una valoración tácita positiva de lo que es y representa el consumo.
Porque hasta los mismos padres dan la autorización para que nosotros lo hagamos; la autorización no es decir tú puedes tomar alcohol cuando quieras, simplemente con un gesto o una acción, la mayoría de veces los padres que suelen tomar trago cuando están bajo el efecto del alcohol, suelen inducir a los hijos a que también ingieran el alcohol. (Grupo focal de adolescentes, Barranquilla)

El consumo de alcohol es un hecho transgeneracional, cuya transmisión "natural" se hace por medio del ejemplo de inducción al consumo por parte de padres, tíos, hermanos mayores y, en ocasiones, abuelos, por ello los adolescentes ven la ingesta de alcohol como una tradición que se sigue desde la adolescencia. En contraste, los padres consideraron que han dado ejemplo a sus hijos, sobrinos $\mathrm{u}$ otros menores de edad, de consumo moderado o abstención, especialmente en Bogotá. Los estudiantes de Medellín fueron quienes más consideraron esta situación.

Yo trabajaba en construcción con mi abuelo y él me ofreció una cerveza y yo dije ¡bueno!, ¡hágale pues! y desde ahí me gustó y entonces seguí tomando desde los 12 años prácticamente. (Grupo focal de adolescentes, Cali).

$[\ldots]$

Mi papá decía: usted ya va a ser mayor de edad, eso no le va a pasar nada, hágale y ya. (Grupo focal de adolescentes, Cali)

Los niños menores de 12 años fueron vistos por los adolescentes como menores de 
edad que no pueden consumir alcohol, mientras a sí mismos no se percibieron así. Contaron historias de inducción del consumo a estos menores de 12 años por parte de los abuelos o los tíos. Esta diferencia que establecieron con los menores de cierta edad, aun cuando para ellos mismos fuera ilegal el consumo de alcohol, pudo observarse más claramente en la categoría de normas y valores.

Por ejemplo en un cumpleaños, si están tomando, a los niños no los acuestan, ni los retiran, ni les piden que se alejen, ni nada, sino que siempre están ahí, pero no están ahí pendientes de lo que está haciendo uno, sino que ellos están jugando, entonces cuando están por ahí como muy cerquita, mi abuelito es el que a veces les dice venga mijo, tómese un poquitico de esto y verá que usted va a ver que eso no es nada, ellos lo prueban y hacen caritas y dicen. ¡ $U y$, no, eso tan maluco! (Grupo focal de adolescentes, Medellín)

Otros adolescentes mencionaron que era común ingerir licor con la familia, ya fuera por la celebración de una fecha especial o por el simple hecho de estar reunidos; por lo general, con la participación de la familia extensa, en la que están presentes abuelos, tíos y primos. En estas ocasiones, el licor es el principal elemento para divertirse y compartir, es el componente articulador de las relaciones familiares en los ratos de ocio y celebración, sin el cual no puede considerarse un momento de fiesta; de no haber, la reunión se acabaría pronto, el compartir estaría limitado a una experiencia más corta y, como dijo un estudiante, "menos profunda". Cada miembro de la familia tiene su ritmo, lo que importa es que no dejen de tomar, pues una vez que se acaban las botellas, se acaba la fiesta, el momento de reunión y la excusa para compartir.

Pues en mi casa se toma en los diciembres, cuando juega la selección Colombia y en algunos cumpleaños de tíos. (Grupo focal de adolescentes, Cali)

$[\ldots]$

Pero uno va más allá de la tradición, siempre las familias colombianas, voy a decir así, siempre toman, y eso es lo que nosotros vemos como normal, y entonces siempre me va a parecer normal porque siempre en diciembre toman [...] o tomamos también, porque no puedo decir que yo no. (Grupo focal de adolescentes, Medellín)

Tanto las actitudes favorables hacia el consumo de alcohol, como las conductas de adultos y pares, inducen al consumo, lo que lo convierte en una actividad social que hace parte de las tradiciones familiares y culturales de los grupos sociales. Las fiestas, paseos, cumpleaños, reuniones por ocasiones o fechas especiales, $\mathrm{y}$, en general, cualquier celebración o evento social que implique compartir con otras personas, son situaciones asociadas con el consumo de licor, y aunque generalmente se hace con la familia, no necesariamente el consumo está únicamente ligado con ella, también lo está con los amigos.

\section{Del dicho al hecho hay mucho trecho: creencias sobre el alcohol}

Las creencias que tienen las familias sobre el consumo de alcohol son diversas; en muchas ocasiones, esas creencias se han 
transmitido a los hijos, y ellos tienen las mismas ideas o se han alimentado con las de otros compañeros. Entre las creencias más comunes y que limitan el consumo de alcohol se encuentran las relacionadas con las consecuencias del uso de licor y los riesgos que genera en la salud física y mental.

Entre las creencias más destacadas para padres y adolescentes estuvo el hecho de que se toma por presión o influencia de pares, o incluso de familiares; los adolescentes manifestaron que no necesariamente ellos han querido tomar, sino que el hecho de encontrarse en entornos en los que se toma y por el ofrecimiento de las personas que los acompañan, ellos han decidido consumir alcohol, asociado con que es "normal tomar en celebraciones".

A mi hijo le están afectando son las amistades que tiene él, hay unas amistades que sí, otras que no y usted sabe que las amistades de hoy en día se dejan llevar por todo, se cuenta todo, se dejan influir. (Grupo focal de padres, Bogotá)

\section{$[\ldots]$}

Bueno, a mí tomar no me gusta, pero yo creo que la gente lo hace como por el ambiente, por los amigos, uno piensa que tomar así es para pasarla bueno y si no lo hace van a pensar mal. (Grupo focal de adolescentes, Medellín)

Otra creencia muy fuerte que se reveló en los grupos focales, especialmente de Bogotá y Cali, fue que ingerir alcohol es un elemento de la cultura que se ha regularizado en las celebraciones; de esta manera, las familias han contribuido a difundir la práctica del consumo de alcohol como una tradición que se transmite culturalmente.

Yo pienso que eso hace más como parte de la cultura, porque toda la vida, que para celebrar siempre hay alcohol $[. .$. y todo se celebra con trago, todo con trago. (Grupo focal de padres, Cali)

\section{$[\ldots]$}

No se podía hacer ninguna celebración sin licor en mi familia. (Grupo focal de padres, Bogotá).

Tanto los padres como los adolescentes consideraron los daños para la salud como una de las principales consecuencias que conlleva el consumo de alcohol en menores de edad. Ambos grupos establecieron diferencias entre las consecuencias inmediatas, como el guayabo y el malestar general, y las consecuencias a largo y mediano plazos. Los adolescentes no tenían muy claros los daños a los que se referían los adultos cuando decían que el alcohol afectaba la salud.

Boté las zapatillas nuevas, ahí sí quede vuelto nada, cuando llegué a la casa una vomitadera y al otro día un guayabo feo. (Grupo focal de adolescentes, Cali)

$[\ldots]$

Va a dañar las neuronas, va a dañar la concentración, o sea se va a hacer un daño grandísimo. (Grupo focal de padres, Medellín)

$[\ldots]$ 
Que es malo, porque sé que en la salud eso produce cosas, pero no sé qué tipo de cosas. (Grupo focal de adolescentes, Barranquilla)

Los adultos no encontraron diferencias entre las consecuencias de menores y mayores de edad; por el contrario, los adolescentes encontraron más diferencias entre adultos y niños.

Sí hay muchas consecuencias, para el hígado, cirrosis, el hígado se les vuelve nada, el cerebro también. (Grupo focal de padres, Bogotá)

\section{$[\ldots]$}

Yo digo que las consecuencias son en el metabolismo del joven; como es diferente al de una persona adulta, pues yo creo que las consecuencias van a ser cada vez peores y van a hacer más daños. (Grupo focal de adolescentes, Cali)

\section{$[\ldots]$}

En la parte de la salud, sé que tomar a temprana edad puede impedir parte del desarrollo del cuerpo, tanto físico como mental, puede tener problemas a largo plazo, o sea, problemas que no tenemos, pero que por tomar los tenemos. (Grupo focal de adolescentes, Barranquilla)

Al hablar de riesgos, los adolescentes pusieron en primer lugar la violencia y las peleas que desencadena el consumo de alcohol, y ningún aspecto vinculado con la salud, como sí lo hicieron los adultos. Expresaron que, en muchas ocasiones, debido a los tragos, la gente hace cosas de las que no es muy consciente o luego se arrepiente, como propiciar peleas y generar conflicto, en ocasiones con los mismos familiares. Esta creencia está fundamentada en sus propias experiencias, y en muchas ocasiones sirve como obstaculizador de consumo. Los adolescentes también ubicaron el ser víctimas de robos y accidentes en el primer lugar en riesgos por el abuso de alcohol, al igual que la violencia y las peleas. Los adultos, así mismo, encontraron que esto era un riesgo, pero no lo ubicaron en un lugar tan relevante como los adolescentes.

Lo que no me gustó fue eso, porque fue la primera vez que hubo pelea en toda la familia y la causa fue el alcohol. (Grupo focal de adolescentes, Cali)

$$
[\ldots]
$$

Estaba con los amigos y se pusieron a tomar los cuatro y iquién sabe qué le echarían porque les robaron todo! y sus supuestos amigos lo dejaron solo. (Grupo focal de padres, Bogotá)

La tradición cultural que supone tomar alcohol parece ser una de las creencias con más peso que promueve el consumo de alcohol en menores de edad, combinado con la influencia que ejerce el entorno de fiesta y celebración que siempre va a acompañado de bebidas alcohólicas. Ampliar la mirada a largo plazo es una de las creencias que más contrarresta el consumo de alcohol, más allá del planteamiento de las consecuencias inmediatas que genera la bebida, como el guayabo, el malestar y las peleas, sino, también, las secuelas físicas y las rupturas familiares que puede provocar el consumo excesivo de esta sustancia. 


\section{¿Y las normas y los valores para qué?}

Esto hace referencia a los parámetros de conducta y a los ideales que pueden favorecer o impedir el consumo de alcohol en los jóvenes. En muchos de estos adolescentes, las normas y valores hacen que se sostenga la tradición de uso de bebidas embriagantes desde edades cada vez más tempranas.

La ausencia de normas respecto al consumo de alcohol para los menores de edad fue una expresión constante en varias regiones, y los adolescentes lo han interpretado como aceptación, por parte de los adultos, del uso de alcohol. En contraste, en los grupos focales de los padres, la principal regla fue que ni siquiera en reuniones familiares un menor de edad podía tomar, particularmente en Barranquilla y Cali. Esto demuestra la contradicción entre las palabras y los hechos, y la necesidad de establecer límites claros para los adolescentes. "En mi familia es muy normal, el que quiera tomar, toma y ya" (Grupo focal de adolescentes, Bogotá). "Lo que decía el papá era que nada de alcohol, aquí en la casa nadie va a tomar porque es malo, esas son las normas, ellos saben" (Grupo focal de padres, Cali).

Algunos adolescentes no consideraron que la norma de no consumir alcohol mientras fueran menores de edad estuviese dirigida a ellos, pues cuando se refirieron a esta regla, consideraron que era para "los niños pequeños", menores de 12 o de 15 años. Los adultos reafirmaron esta idea, al no poner, en la mayoría de los casos, restricciones explícitas al consumo de menores de 18 años.

En mi familia, después de que uno cumpla los 15, ya está supuestamente capacitado para tomar y para estar en las fiestas todo el tiempo, toda la noche o todo el día. (Grupo focal de adolescentes, Barranquilla)

[...]

La norma es esa, que los niños hasta determinada hora estén con uno y que tampoco estén cuando se está tomando. (Grupo focal de adolescentes, Medellín)

Respecto a los valores, los estudiantes indicaron que la unión familiar y el seguimiento de la cultura y la tradición han favorecido el uso de bebidas embriagantes. Esto implica que los adolescentes normalicen el consumo de alcohol desde muy pequeños, porque es algo que hace "todo el mundo" en "todo momento".

Mi familia también se reúne, pues es muy unida, y un cumpleaños, Halloween, diciembre, todos se reúnen en el patio, siempre van es a mi casa por mi abuela, y también toman mucho, les gusta mucho el guaro y el tequila y bueno, a mí también. (Grupo focal de adolescentes, Medellín)

\section{$[\ldots]$}

Como dice mi compañero, un promotor de consumo es la tradición, uno toma por tradición. (Grupo focal de adolescentes, Medellín)

Sin embargo, algunos valores interiorizados por algunos estudiantes son obstaculizadores del consumo de alcohol: la responsabilidad es uno de ellos, al momento de decidir no tomar. Refirieron acciones a corto plazo, 
sobre todo relacionadas con los deberes del colegio; otros expresaron planes a largo plazo, como estudiar para las pruebas del Estado y formarse profesionalmente. "No tomo cuando tengo que hacerme responsable de algo, como por ejemplo, cuando hay exámenes" (Grupo focal de adolescentes, Cali).

La responsabilidad y la visión a largo plazo fueron valores que los adolescentes ubicaron como prioritarios al momento de decidir no tomar. En contraste, los valores que promueven el consumo de alcohol fueron la unión familiar y el seguimiento de la tradición y la cultura.

\section{Discusión Y CONCLUSIONES}

A partir de los grupos focales, es posible determinar que el consumo de alcohol es un hecho social, que se realiza la mayoría de las ocasiones en el entorno familiar y en el de pares (Kloep, Ingerbrigsten, Glendinning y Espness, 2001; Yoon, Lam, Sham \& Lam, 2015), incluso los primeros tragos se dan en el contexto de la familia, pese al reconocimiento de riesgos y consecuencias negativas. Al ser una actividad que se sigue por socialización, las dinámicas familiares en cuanto a normas, límites, autoridad, socialización y relaciones son fundamentales para los adolescentes a la hora de tomar la decisión de ingerir alcohol o no (Torres, Ortega, Garrido \& Reyes, 2008).

La ausencia de normas claras respecto al consumo de alcohol es interpretada por los adolescentes como aceptación de uso de alcohol por parte de los adultos, lo cual es muy coherente con lo expresado por Gossrau-Breen, Kuntsche y Gmel (2010), quienes afirman que el ejemplo de consumo es más determinante que el establecimiento de normas en el hogar.

Parecería que en nuestro medio, cada celebración o evento familiar o social lleva asociado el consumo de licor; este es el principal elemento para compartir y articular las relaciones sociales, sin el cual no puede considerarse un momento de fiesta. Se permite, entonces, que los adolescentes ingieran algunas cantidades. Tal como lo mencionan Flórez y Trujillo (2013), la decisión de consumir depende en gran parte de la situación en la que se encuentre el joven; es decir, el entorno influye considerablemente en el comportamiento de los adolescentes frente al consumo de alcohol, y no necesariamente lleva implícitos procesos reflexivos. Que consumir alcohol sea un valor social normalizado en la cultura genera entornos propicios para la ingesta temprana de licor.

La normalización del consumo de alcohol es un hecho social, que refleja los patrones y costumbres de la comunidad de pertenencia de los adolescentes; así, la normalización también es definida por las historias de consumo familiar, en cuanto a frecuencia de uso, cantidad ingerida, aceptación/rechazo de la sustancia y lugar de consumo. Los resultados indicaron que hay una historia de uso de alcohol en las familias, ya sea en el núcleo familiar o en la familia extensa, y esta actitud positiva es tomada por los adolescentes como la posibilidad de ingerir bebidas embriagantes siendo menores de edad.

En reuniones familiares consumen algunas cantidades de alcohol, en ocasiones toman mínimas dosis; en otras, siguen el mismo ritmo de los adultos. Esta observación se acerca a lo encontrado por Becoña (2002), 
quien sostiene que las actitudes favorables de los adolescentes son formadas en la socialización con los padres, otros familiares o el grupo social, y esto podría incrementar la probabilidad de consumo por parte de menores de edad.

La familia ha sido un factor determinante para el consumo de alcohol en adolescentes, ya que han inculcado a sus hijos que ingerirlo es algo normal en el proceso de vida y que la intensidad depende principalmente de la edad, por tanto, es más válido el alto consumo en edades más tempranas, como ellos mismos lo han experimentado y como la literatura también lo expresa. Así lo plantea el estudio de Valentine et al. (2010), en Reino Unido; allí se encontró que los jóvenes adoptan patrones de consumo de alcohol similares a los de sus padres en su propia juventud, y que las dinámicas actuales promueven un mayor consumo que en los años ochenta del siglo pasado.

En Colombia, la tradición cultural que supone consumir licor parece ser una de las creencias con más peso que promueve el uso de alcohol en menores de edad, combinado con la influencia que ejerce el entorno de fiesta y celebración que siempre va acompañado de bebidas alcohólicas, como también lo encontraron Flórez y Trujillo (2013). La unión familiar y el seguimiento de la cultura y la tradición son elementos que han favorecido el uso de bebidas embriagantes en los adolescentes del estudio, y son factores que Gmel, Kuntsche y Rehm (2011) proponen como promotores del consumo de alcohol en adolescentes.

Después de este análisis, es posible concluir que el consumo de alcohol por parte de menores de edad es un hecho social normalizado por la cultura, en que la tradición de uso de bebidas embriagantes se transmite de generación en generación, y los propios hogares y casas de familiares suelen ser los espacios en los que los menores de edad inician su consumo.

Las tradiciones familiares desempeñan un rol importante en la promoción del consumo, y hacen referencia a acciones que van desde la legitimación del uso de alcohol, en algunas ocasiones a lo largo del año, especialmente en fiestas decembrinas, hasta el consumo en cualquier reunión familiar, lo que adjudica valor a la unión familiar, una de las razones principales para tolerar el consumo entre menores de edad.

Estas tradiciones familiares de consumo y los valores que las promueven se suman a las creencias de adultos y adolescentes, quienes consideran que la presión o influencia de pares, de familiares o del entorno aumentan la oportunidad de iniciarse tempranamente en el consumo de alcohol, así como de mantenerlo a lo largo de la vida. Los daños en la salud y los riesgos sociales que consideraron los participantes no fueron particularmente importantes al momento de tomar la decisión de ingerir alcohol, lo que supone que las creencias respecto al consumo de alcohol ocupan un lugar secundario, en comparación con las tradiciones y ejemplo de adultos significativos.

Con base en la literatura sobre el tema, la experiencia de la autora y los resultados de este estudio, también es posible concluir que la ineficacia de los programas preventivos se debe, en parte, a que están dirigidos a fortalecer las habilidades de rechazo, asertividad, toma de decisiones o pensamiento crítico de los adolescentes en su entorno 
escolar y de pares, y no tienen en cuenta aspectos familiares, como las tradiciones y los valores que hacen que sus primeros consumos se den en el hogar. Por ser los miembros de la familia figuras de autoridad para los menores de edad, las habilidades estudiadas en el ámbito escolar pierden fuerza a la hora de aplicarlas, pues fueron principalmente diseñadas para ser puestas a prueba con otros jóvenes, y no con adultos.

Dado que la familia es uno de los espacios por excelencia en que se presenta consumo de alcohol en adolescentes, se hace necesario formarlas y reenfocar sus dinámicas de consumo, de modo que se otorgue la primera responsabilidad de supresión del uso de bebidas embriagantes a los adultos, y no a los adolescentes. De esta manera, se podría contribuir a cambiar la tradición, a generar más extrañeza y menos normalización del uso de alcohol en menores de edad, y a modificar las creencias relacionadas con la promoción del consumo en los valores familiares.

\section{Agradecimientos}

La autora agradece al doctor Roberto Suárez, profesor de la Universidad de los Andes, por la dirección del presente trabajo y la orientación desde el aspecto antropológico, y al doctor Augusto Pérez, director de la Corporación Nuevos Rumbos, por sus aportes y sugerencias.

\section{REFERENCIAS}

Arthur, M. W., Hawkins, J. D., Pollard, J. a., Catalano, R. F., \& Baglioni Jr., a. J. (2002). Measuring Risk And Protective Factors For Substance Use, Delinquency, And
Other Adolescent Problem Behaviors: The Communities That Care Youth Survey. Evaluation Review, 26(6), 575-601.

Barker, J. C., \& Hunt, G. (2004). Representations of family: A review of the alcohol and drug literature. International Journal of Drug Policy, 15, 347-356.

Becoña, E. (2002). Bases científicas de la prevención de las drogodependencias. Madrid: Ed. Ministerio del interior. Plan nacional sobre drogas.

Bernard, H. R. (2011). Research methods in anthropology: qualitative and quantitative approaches. Estados Unidos: Altamira Press.

Briones, T. L., \& Woods, J. (2013). Chronic binge-like alcohol consumption in adolescence causes depression-like symptoms possibly mediated by the effects of BDNF on neurogenesis. Neuroscience, 254, 324-334.

Campos, H. (2007). Diccionario de Sociología. Recuperado de http://ciberconta. unizar.es/leccion/sociodic/tododic.pdf

Fagan, A. A., Van Horn, M. L., Hawkins, J. D., \& Arthur, M. (2007). Using community and family risk and protective factors for community-based prevention planning. Journal of Community Psycho$\log y, 35(4), 535-555$.

Flórez, I. A., \& Trujillo, A. M. (2013). ¿ Tomar no tomar ?: análisis de los motivos de consumo y no consumo de alcohol en jóvenes * To Drink or not to Drink?: Analysis of Alcohol Consumption and non Consumption Reasons in Adolescents Resumen Introducción. Diversitas, 9(1), 199-215.

Gmel, G., Kuntsche, E., \& Rehm, J. (2011). Risky single-occasion drinking: Bingeing is not bingeing. Addiction, 106, 1037-1045. 
Gossrau-Breen, D., Kuntsche, E., \& Gmel, G. (2010). My older sibling was drunk - Younger siblings' drunkenness in relation to parental monitoring and the parent-adolescent relationship. Journal of Adolescence, 33(5), 643-652.

Guelbenzu, R. (2011). Pequeña historia del alcohol. Recuperado de http://www.winedefender.org/?p=927

Khoddam, R., Worley, M., Browne, K. C., Doran, N., \& Brown, S. A. (2015). Family history density predicts long term substance use outcomes in an adolescent treatment sample. Drug and Alcohol Dependence, 147, 235-242.

Kloep, M., Ingerbrigsten, J., Glendinning, A., \& Espness, G. (2001). Young People in Drinking Societies? Norwegian Scottish and Swedish Adolescents' Perception of Alcohol Use. Health Education Research, 16, 279-291.

Lyman, M. D. (2011). Drugs in society: causes, concepts and control. Burlington, MA: Anderson Publishing.

Ministerio de Justicia y el Derecho, Ministerio de Educación, Ministerio de Salud y Protección Social, UNODC \& Organización de Estados Americanos. (2011). Estudio nacional de consumo de sustancias psicoactivas en poblacion escolar Colombia - 2011 1. Bogotá, D. C.

Mora, J. \& Natera, G. (2001). Expectativas, consumo de alcohol y problemas asociados en estudiantes de la ciudad de México. Salud Pública de México, 43(2).

Pascual, M., Pla, A., Miñarro, J., \& Guerri, C. (2014). Neuroimmune activation and myelin changes in adolescent rats exposed to high-dose alcohol and associated cognitive dysfunction: A review with reference to human adolescent drinking. Alcohol and Alcoholism, 49(2), 187-192.

Pérez-Gómez, A. (1986). Cocaína: surgimiento y evolución de un mito. Bogotá: Catálogo Científico.

Pérez-Gómez, A., Mejía-Trujillo, J., Reyes-Rodríguez, M. \& Cardozo, F. (2015). Consumo de alcohol en menores de 18 años en Colombia: 2015. Bogotá: Corporación Nuevos Rumbos.

Pérez-Gómez, A., Ruiz-Giraldo, J., Villar-Concha, E., \& Uribe-Martínez, T. (2000). Principales sustancias psicoactivas y sus efectos. En A. Pérez-Gómez (Ed.), El libro de las drogas: manual para la familia (pp. 45-117). Bogotá: Presidencia de la República de Colombia.

Pérez-Gómez, A., \& Scoppetta, O. (2009). Consumo de alcohol en menores de 18 años en Colombia: 2008 un estudio con jóvenes escolarizados de 12 a 17 años en 7 capitales de departamento y dos municipios pequeños. Bogotá: Corporación Nuevos Rumbos. Recuperado de http:// scholar.google.com/scholar?hl=en\&bt$\mathrm{nG}=$ Search\&q=intitle:Consumo+de+alcohol+en+menores+de+18+años+en+Colombia+:+2008+un+estudio+con+jóvenes+escolarizados $+\mathrm{de}+12+\mathrm{a}+17+\mathrm{a}-$ nos+en+7+capitales+de+departamento $+y+d o s+$ municipios+pequeños $\# 0$

Pérez-Gómez, A., Scoppetta, O., \& Flórez-Alarcón, L. (2011). Age at onset of alcohol consumption and risk of problematic alcohol and psychoactive substance use in adulthood in the general population in Colombia. The Journal of International Drug, Alcohol and Tobacco Research, 1, 19-24.

Rolando, S., Beccaria, F., Tigerstedt, C., \& Törrönen, J. (2012). First drink: What does it mean? The alcohol socialization 
process in different drinking cultures. Drugs: Education, Prevention, and Policy, 19(19), 201-212.

Rowland, B., Toumbourou, J. W., Satyen, L., Livingston, M., \& Williams, J. (2014). Addictive Behaviors The relationship between the density of alcohol outlets and parental supply of alcohol to adolescents. Addictive Behaviors, 39(12), 1898-1903.

Schofield, T. P., \& Denson, T. F. (2013). Alcohol outlet business hours and violent crime in New York State. Alcohol and Alcoholism, 48(3), 363-369.

Scholte, R. H. J., Poelen, E. a P., Willemsen, G., Boomsma, D. I., \& Engels, R. C. M. E. (2008). Relative risks of adolescent and young adult alcohol use: The role of drinking fathers, mothers, siblings, and friends. Addictive Behaviors, 33, 1-14. Sierra, D., Pérez Trujillo, M., Pérez Gómez, A. y Muñoz, M. (2005). Representaciones sociales en jóvenes consumidores y no consumidores de drogas. Adicciones, 17(4), 349-360.

Stickley, A., Koyanagi, A., Koposov, R., Razvodovsky, Y., \& Ruchkin, V. (2013). Adolescent binge drinking and risky health behaviours: Findings from northern Russia. Drug and Alcohol Dependence, 133(3), 838-844.

Stimson, G. V. (2006). El consumo de alcohol en contexto: Responsabilidad de todos. Recuperado de http://www.icap.org/ LinkClick.aspx?fileticket=LKiN80N6Y$\mathrm{Go}=\&$ tabid $=76$

Torres, L., Garrido, A., Reyes, A., \& Ortega, P. (2008). Responsabilidades en la crianza de los hijos. Enseñanza e investigación en Psicología, 13(1), 77-89.

Vaillant, G. (1983). The natural history of alcoholism. Cambridge, MA: Harvard University Press.

Valentine, G., Holloway, S. L., \& Jayne, M. (2010). Generational patterns of alcohol consumption: Continuity and change. Health E Place, 16(5), 916-925.

Ward, R. J., Lallemand, F., \& De Witte, P. (2014). Influence of adolescent heavy session drinking on the systemic and brain innate immune system. Alcohol and Alcoholism, 49(2), 193-197.

Yoon, S., Lam, W. W. T., Sham, J. T. L., \& Lam, T.-H. (2015). Learning to drink: How Chinese adolescents make decisions about the consumption (or not) of alcohol. International Journal of Drug Policy. doi:10.1016/j.drugpo.2015.09.001 